\title{
A prospective longitudinal study of the prevalence of post-traumatic stress disorder resulting from childbirth events
}

\author{
K. L. Alcorn ${ }^{1}$, A. O’Donovan ${ }^{1}$, J. C. Patrick ${ }^{2}$, D. Creedy ${ }^{3}$ and G. J. Devilly ${ }^{1,4 *}$ \\ ${ }^{1}$ School of Psychology, Griffith University, Queensland, Australia \\ ${ }^{2}$ Department of Psychology, University of Southern Queensland, Queensland, Australia \\ ${ }^{3}$ Griffith Health, Griffith University, Queensland, Australia \\ ${ }^{4}$ Griffith Institute for Health \& Medical Research, Griffith University, Queensland, Australia
}

Background. Childbirth has been linked to postpartum impairment. However, controversy exists regarding the onset and prevalence of post-traumatic stress disorder (PTSD) after childbirth, with seminal studies being limited by methodological issues. This longitudinal prospective study examined the prevalence of PTSD following childbirth in a large sample while controlling for pre-existing PTSD and affective symptomatology.

Method. Pregnant women in their third trimester were recruited over a 12-month period and interviewed to identify PTSD and anxiety and depressive symptoms during the last trimester of pregnancy, 4-6 weeks postpartum, 12 weeks postpartum and 24 weeks postpartum.

Results. Of the 1067 women approached, 933 were recruited into the study. In total, 866 (93\%) were retained to 4-6 weeks, $826(89 \%)$ were retained to 12 weeks and $776(83 \%)$ were retained to 24 weeks. Results indicated that, uncontrolled, $3.6 \%$ of women met PTSD criteria at $4-6$ weeks postpartum, $6.3 \%$ at 12 weeks postpartum and $5.8 \%$ at 24 weeks postpartum. When controlling for PTSD and partial PTSD due to previous traumatic events as well as clinically significant anxiety and depression during pregnancy, PTSD rates were less at $1.2 \%$ at $4-6$ weeks, $3.1 \%$ at 12 weeks and $3.1 \%$ at 24 weeks postpartum.

Conclusions. This is the first study to demonstrate the occurrence of full criteria PTSD resulting from childbirth after controlling for pre-existing PTSD and partial PTSD and clinically significant depression and anxiety in pregnancy. The findings indicate that PTSD can result from a traumatic birth experience, though this is not the normative response.

Received 15 September 2009; Revised 18 November 2009; Accepted 21 November 2009; First published online 11 January 2010

Key words: Childbirth, prevalence, postnatal, trauma.

\section{Introduction}

Evidence suggests that childbirth is an event that could be psychologically traumatic, leading to the development of post-traumatic stress disorder (PTSD) and subsyndromal PTSD (Wijma et al. 1997; Olde et al. 2005). A traumatic birth can interfere with a mother's attachment to her infant and her overall adjustment (Ballard et al. 1995; Allen, 1998; Soet et al. 2003; Beck, $2004 a)$, negatively affect the relationship with her partner (Fones, 1996; Allen, 1998; Beck, 2004a) and overshadow decisions about having additional children (Fones, 1996; Saisto et al. 2001; Bailham \& Joseph,

* Address for correspondence: Dr G. J. Devilly, School of Psychology and Griffith Institute for Health \& Medical Research, Griffith University, Mt Gravatt, Qld 4122, Australia.

(Email: grant@devilly.org)

(Email: a.odonovan@griffith.edu.au)
2003; Ryding et al. 2003; Beck, 2004b). Aside from the impact on the mother and her family, traumatic birth could impact negatively on the healthcare system by increasing use of medical services (Switzer et al. 1999) by both the parents and the offspring.

Studies have suggested a prevalence rate of PTSD following birth from $1 \%$ to $6 \%$ (Creedy et al. 2000; Ayers \& Pickering, 2001). However, aside from the debate regarding the broadening of the conceptual boundaries of what qualifies as a traumatic event (Baldwin et al. 2004), drawing firm conclusions about the prevalence of PTSD resulting from childbirth is difficult due to a number of methodological shortcomings (Johnston-Robledo \& Barnack, 2004). For example, there have only been two prospective, longitudinal studies of PTSD resulting from childbirth. In the seminal study by Ayers \& Pickering (2001), there was a relatively small sample $(n<300)$ 
and no assessment for pre-existing PTSD using diagnostic criteria before the birthing event. In the second study, Söderquist et al. (2006) assessed a large number of prospective mothers with a longitudinal design and also assessed fear of birth during pregnancy. This study was a subset of Wijma et al. (1997) and found a $3 \%$ trauma rate (assessing symptomatic criteria B, C and D) at some point up to 11 months postpartum. The authors suggested that the low rate may be due to birth on the whole being considered a positive experience, yet noted that negative birthing events can lead to PTSD. Major drawbacks of this study, however, included the utilization of a non-validated measure of PTSD, not using DSM-IV criteria to assess for 'trauma' (criterion A could be met if the mothers found the birthing experience as merely 'trying'), no assessment of pre-existent PTSD and the assessment of functional impairment at an individual symptom level rather than a diagnostic level.

The present study is the first large, prospective, longitudinal study assessing the full diagnostic criteria for PTSD following childbirth while also controlling for pre-existing PTSD and affective symptomatology. The potential confound of having a diagnosis of PTSD from any event prior to birth has not been examined systematically nor has the possible contribution of other antenatal factors and mood disorders on the subsequent development of birth-related PTSD (Bailham \& Joseph, 2003). This prevents a determination of whether the observed PTSD symptoms developed from a childbirth event or are an expression of pre-existing disequilibria (Ayers \& Pickering, 2001).

Further, without assessing the traumatic nature of the birth event, it is also unknown whether postpartum PTSD symptoms are truly reflective of PTSD or reflective of 'trauma-like symptoms'. This is particularly relevant given that PTSD, anxiety and depression share symptomatic expressions of general distress (Engelhard et al. 2003). With debate on whether birthing events can even lead to the development of PTSD, the purpose of this article is to report accurate prevalence rates for PTSD caused by birthing events, rather than present a model explaining these rates.

It is notable that few studies have used established measures of PTSD (Ayers \& Pickering, 2001), an omission when investigating a disorder (Foa \& Meadows, 1997). Furthermore, while there are a number of single case studies reported in the literature (e.g. Ballard et al. 1995; Fones, 1996) and several studies with a small sample size (i.e. <200, e.g. Allen, 1998; Keogh et al. 2002; Soet et al. 2003), the relatively low life-time prevalence rate of PTSD would indicate that a large intake sample is necessary.
Finally, there is a need to consider the prevalence of diagnosable PTSD as distinct from the prevalence of women who have subclinical symptoms (i.e. partial PTSD). There is also evidence that the prevalence of partial PTSD is much higher than PTSD and that subsyndromal PTSD is associated with significant disability (Ballenger et al. 2000), functional impairment (Stein et al. 1997) and adjustment postpartum (Soet et al. 2003). The present study builds upon previous influential research by investigating the development of PTSD and subclinical symptoms in a large cohort of women recruited in their third trimester of pregnancy. In order to track the development of PTSD and related symptoms, women were followed up at three time points : 4-6 weeks; 12 weeks; 24 weeks postpartum.

\section{Method}

\section{Sample and procedure}

The protocols used in this study were approved by all relevant university and hospital ethics committees. A sample of women was recruited from two antenatal clinics in Brisbane, Australia, and from two adjoining regional antenatal clinics. Researchers approached consecutive attendees who were waiting for their scheduled antenatal appointments. Women were eligible to participate if they were at least 18 years of age, in their third trimester of pregnancy (from 28 weeks gestation), able to read and write in English and contactable by phone.

Of the 1067 women approached, 933 agreed to participate during the last trimester of pregnancy $(87 \%$ response rate). A total of $93 \%(866 / 933)$ of these women completed at 4-6 weeks postpartum, $89 \%$ (826/933) completed at 12 weeks postpartum and $83 \%$ (776/933) completed at 24 weeks postpartum. The 134 non-responders (those who declined to take part in the research) did, nonetheless, answer a short questionnaire relating to their age, marital status, length of relationship, number of children, educational level and ethnic status. There were no significant differences $(p<0.01)$ between responders and non-responders on these variables except for ethnic status. Asian women were less likely to agree to the research compared with Caucasian/Northern European women $\left[\chi^{2}(10)=41.68\right.$, $p<0.001]$. The attrition rate between first and last assessment was significant in that those who dropped out were more likely to belong to a lower socioeconomic status (SES) and display more pathology at intake.

The following demographics are given to gauge the representativeness of our sample, with Australian national data (AD) following each statistic. As can be seen, and would be expected with such a large group, 
our sample is quite consistent with Australian data. The mean age of participants was 29 years (mean = 28.56, S.D. $=5.64$; AD mean $=29.8$; Laws \& Hilder, $2008)$, most women were married $(53.9 \%)$ or cohabitating $(30.1 \% ; \mathrm{AD}=82.1 \%$ combined; Social Policy Research Centre, 2006), some were single (13.5\%) and a small percentage were separated $(1.9 \%)$ or divorced $(0.5 \% ; \mathrm{AD}=14.6 \%$ combined; Social Policy Research Centre, 2006). The average relationship length was 6 years. Approximately $86 \%$ were Caucasian $(\mathrm{AD}=89.1 \%$; Social Policy Research Centre, 2006), with remaining participants reflecting a diverse ethnic background. Modal educational level was just less than high school completion (grades 10 or $11 ; 24.7 \%$ ), followed by high school completion $(22.8 \%)$, completion of a technical and further education certificate $(20.4 \%)$, participation in tertiary studies (1-4 years; $18.4 \%)$, completion of a tertiary higher degree $(7.6 \%)$ and less than grade 10 high school completion (6.2\%). Most women had a gross annual household income between AUS $\$ 36000$ and $\$ 50000$ (23\%; AD = median household income for 2005; Australian Bureau of Statistics, 2007) and AUS\$51000-80000 (23.2\%). The majority of women nominated homemaker for their employment status (48.7\%), followed by full $(21.8 \%)$ and part-time $(9.7 \%)$ employment, unemployment $(9.6 \%)$, casual work $(9.1 \%)$, disability pension $(0.8 \%)$ and regular shift work $(0.4 \%)$. More women than not indicated that they already had children [ 577 women $(61.8 \%), \mathrm{AD}=58.4 \%$; Laws \& Hilder, 2008].

\section{Assessment of PTSD and partial PTSD}

The Posttraumatic Diagnostic Scale (PDS self-report; Foa et al. 1997) was selected to assess prior exposure to traumatic events, PTSD symptom severity and PTSD diagnoses. In pregnancy, participants were also asked to specify the event 'that bothers them the most' when the focus was on current PTSD (Stein et al. 1997).

For postpartum PTSD (related only to the birth), women who met criteria A1 and A2 could meet PTSD or partial PTSD criteria. To examine whether or not criteria A1 (a traumatic event) and A2 (fear response during trauma) was met during birth, we followed exact DSM-IV-TR criteria (American Psychiatric Association, 2000). Participants rated stressors on a 10-point scale ranging from 1 (not at all) to 10 (very much), with $\geqslant 2$ representing a 'yes' answer. The assessment of criteria A1 and A2 are central to this article and are, therefore, discussed extensively.

The exact questions asked to assess criterion A1 were: (1) 'Did you feel that your life or your baby's life was threatened during or after the birth?' (2) 'Did you think that you or your baby might die?' (3) 'Did you experience an actual injury or threat of serious injury around the time of the birth?' (4) 'Did your baby experience an actual injury or threat of serious injury around the time of birth?' (5) 'Did you feel worried about your own safety during the labour and childbirth?' (6) 'Did you feel worried about your baby's safety during the labour and childbirth?' (7) 'Were you frightened that your baby was not normal?' Items 1-4 permitted an assessment of 'actual or threatened death or serious injury' (APA, 2000, p. 467). Items 5-7 permitted an assessment of 'a threat to the physical integrity of self or others' (APA, 2000, p. 467). No woman who received a diagnosis of PTSD only endorsed item 7 and the diagnosis of PTSD in only four women relied on one or more of items 5,6 or 7 . If a participant's baby died during the birth, then criterion A1 was met $(n=3,0.3 \%)$. Further, women who did not experience the commencement of labour were instructed to answer the questions that had a reference to labour and childbirth in relation to the birth event itself.

Criterion A2 was assessed through the following item: 'During or immediately after childbirth, did you feel intense fear, helplessness, terror or horror?' As with $\mathrm{A} 1$, the item was rated on a 10 -point scale $(1=$ not at all to $10=$ very much), with $\geqslant 2$ representing an affirmative answer. The PDS also assesses impairment in important areas of functioning. Criterion F for PTSD was fulfilled if participants answered 'yes' on at least one item, thereby indicating impairment in important areas of functioning.

Partial PTSD was diagnosed if the DSM-IV-TR criteria for PTSD were met except that an individual 'lacked one or two of the necessary three criterion C (avoidance) symptoms and/or they lacked one of the necessary two criterion D (hyperarousal) symptoms' (Stein et al. 1997, p. 1116). Based on this definition, three partial PTSD groups were formed: group 1 met all PTSD criteria except for one or two of the necessary three criterion $\mathrm{C}$ symptoms; group 2 met all PTSD criteria except for one of the necessary two criterion D symptoms; group 3 met all PTSD criteria except for one or two of the necessary three criterion C symptoms and one of the necessary two criterion $D$ symptoms. Partial PTSD-like symptom groups met the same criteria as partial PTSD except the criteria for a traumatic birth event were not met (i.e. a non-criterion A event).

The concurrent validity of the PDS was examined against the Clinician Administered PTSD Scale (CAPS), a scale commonly seen as the gold standard for a diagnosis of PTSD (Griffin et al. 2004). A random selection of women $(n=95)$ who completed the study at 24 weeks postpartum also agreed to complete the CAPS. Overall, the PDS and CAPS correlated highly at $r=0.81$ and cross-tabulations indicated good 
agreement between the CAPS and the PDS on all criteria $(\mathrm{A}=77.89 \% ; \mathrm{B}=88.42 \% ; \mathrm{C}=87.36 \% ; \mathrm{D}=$ $78.95 \% ; \mathrm{E}=100 \% ; \mathrm{F}=63.16 \%)$.

\section{Assessment of anxiety}

Clinically significant levels of anxiety in pregnancy and postpartum were assessed with the State-Trait Anxiety Inventory (STAI) forms Y-1 and Y-2 (Spielberger et al. 1983). The STAI has been utilized in research examining PTSD following childbirth (Creedy et al. 2000; Soet et al. 2003), in other PTSD studies (Freedman et al. 2002; Greening et al. 2002) and in studies with pregnant (Sjostrom et al. 2002) and postpartum (Matthey et al. 2003) women. Overall, the STAI has been extensively researched and has acceptable reliability and validity (Spielberger et al. 1983; Ayers, 2001). Women who received total subscale scores $>40$ were considered as having clinically significant symptoms of anxiety (Spielberger et al. 1983).

\section{Assessment of depression}

The Edinburgh Postnatal Depression Scale (EPDS; Cox et al. 1987) was used to assess clinically significant levels of depression in pregnancy and postpartum. The measure has acceptable psychometric properties (Cox et al. 1987; Eberhard-Gran et al. 2001) and has been widely used with pregnant and postpartum women and new mothers (Righetti-Veltema et al. 2002). Consistent with the EPDS recommendations and past research, scores of $\geqslant 12$ were used to indicate possible depression and scores of $\geqslant 14$ were used to indicate probable depression during pregnancy (Murray \& Cox, 1990). Possible depression postpartum was indicated through scores of $\geqslant 9$ and probable depression through scores of $\geqslant 12$ (Matthey, 2004).

\section{Results}

\section{Pre-birth trauma prevalence}

Approximately $91 \%$ of women self-reported experiencing a potentially traumatic event at some point in their life and $43.3 \%$ met DSM-IV criteria for a traumatic event. A considerable number of women reported two $(n=187,22.2 \%)$ or more $(n=448,53.1 \%)$ potentially traumatic events throughout their life.

\section{Prevalence of traumatic births}

A large proportion of this sample reported a 'traumatic' birth event, at 4-6 weeks post-partum, using the DSM-IV-TR criteria $(n=394,45.5 \%)$. The rate of traumatic birth events in women who had not experienced a prior traumatic life event (that met criterion A) was $43.5 \%$. Thus, prior traumatic events did not contaminate the rate of traumatic birth events $\left[\chi^{2}(1)=0.07\right.$, not significant $]$. Of those who endorsed criterion A1 $(n=727 ; 83.95 \%)$, only $54.2 \% \quad(n=394)$ endorsed A2 as well. Of those who endorsed A2 ( $n=$ $429 ; 49.54 \%)$ only $35(8.16 \%)$ had not endorsed A1.

In addition to criteria $\mathrm{A} 1$ and $\mathrm{A} 2$, women were asked whether they experienced any of a series of birth events that were seen as being potentially traumatic. This was to enable us to see which actual events were most related to meeting criteria for PTSD. These events included experiencing an episiotomy, vaginal tear, use of forceps or vacuum instruments to assist vaginal birth, general anaesthetic, emergency Caesarean, baby being placed in a neonatal intensive care unit, baby being born premature, baby experiencing an injury during labour or birth, woman experiencing an injury during labour or birth, woman experiencing a medical complication, baby being born with a medical illness or disability, baby experiencing medical complications and other obstetric complications during birth. Of the 866 women assessed at 1 month postpartum, $80.1 \%$ endorsed one or other of these events. By 12 weeks postpartum, this grew to $80.6 \%$ and $80.7 \%$ by 24 weeks postpartum. Of those women who went on to meet full PTSD criteria at 4-6 weeks postpartum, all had experienced at least one of these birthing events, during which they believed either their life/well-being or the life/well-being of their baby was in danger. But by 12 weeks postpartum, $9.6 \%$ reported not experiencing at least one of these events, with only $4.4 \%$ by 24 weeks postpartum not experiencing one of these birthing events. In other words, unexpected and potentially harrowing birthing events are common, yet do not necessarily account for women developing PTSD. Yet, those women who did develop PTSD invariably experienced such events.

\section{PTSD and partial PTSD postpartum}

Data regarding estimates of PTSD, partial PTSD and PTSD-like symptoms (both of criterion A not met) are presented in Table 1. Note that the rate of PTSD increases from $3.6 \%$ to $6.3 \%$ from $4-6$ weeks postpartum to 12 weeks postpartum, before declining slightly to $5.8 \%$ at 24 weeks postpartum. Following childbirth, $8.9 \%$ of women met criteria for PTSD at least once over the three postpartum assessments. PTSD-like symptoms (i.e. symptoms that mirror PTSD symptoms but result from a non-criterion A event) were not examined during the last trimester of pregnancy because participants were instructed to rate symptom severity only if they had experienced a potentially traumatic event. 
Table 1. Prevalence of PTSD, partial PTSD and PTSD-like symptoms pre-birth (any trauma) and postpartum due to birthing event

\begin{tabular}{|c|c|c|c|c|}
\hline & Pre-birth & $\begin{array}{l}\text { 4-6 weeks } \\
\text { postpartum }\end{array}$ & $\begin{array}{l}3 \text { months } \\
\text { postpartum }\end{array}$ & $\begin{array}{l}6 \text { months } \\
\text { postpartum }\end{array}$ \\
\hline \multicolumn{5}{|l|}{ PTSD } \\
\hline Full PTSD & $75(8.0 \%)$ & $31(3.6 \%)$ & $52(6.3 \%)$ & $45(5.8 \%)$ \\
\hline Partial PTSD group 1 & $21(2.3 \%)$ & $12(1.4 \%)$ & $17(2.1 \%)$ & $14(1.8 \%)$ \\
\hline Partial PTSD group 2 & $10(1.1 \%)$ & $1(0.1 \%)$ & $2(0.2 \%)$ & $1(0.1 \%)$ \\
\hline Partial PTSD group 3 & $9(1.0 \%)$ & $4(0.5 \%)$ & $6(0.7 \%)$ & $2(0.3 \%)$ \\
\hline \multicolumn{5}{|l|}{ PTSD-like symptoms } \\
\hline Full PTSD-like symptoms & N.A. & $4(0.5 \%)$ & $23(2.8 \%)$ & $23(3.0 \%)$ \\
\hline Partial PTSD-like symptoms group 1 & N.A. & $6(0.7 \%)$ & $13(1.6 \%)$ & $5(0.6 \%)$ \\
\hline Partial PTSD-like symptoms group 2 & N.A. & $0(0.0 \%)$ & $0(0.0 \%)$ & $0(0.0 \%)$ \\
\hline Partial PTSD-like symptoms group 3 & N.A. & $0(0.0 \%)$ & $3(0.4 \%)$ & $0(0.0 \%)$ \\
\hline No classification & $818(87.7 \%)$ & $808(93.3 \%)$ & $710(86.0 \%)$ & $686(88.4 \%)$ \\
\hline Total & $933(100 \%)$ & $866(100 \%)$ & $826(100 \%)$ & $776(100 \%)$ \\
\hline
\end{tabular}

Partial post-traumatic stress disorder (PTSD) group 1 met all PTSD criteria except for one or two of the necessary three criterion C symptoms; group 2 met all PTSD criteria except for one of the necessary two criterion D symptoms; group 3 met all PTSD criteria except for one or two of the necessary three criterion C symptoms and one of the necessary two criterion D symptoms. Partial PTSD-like symptom groups met the same criteria as partial PTSD except the criteria for a traumatic birth event were not met (i.e. a non-criterion A event).

\section{Adjusted PTSD, partial PTSD and PTSD-like symptoms postpartum}

Estimates of PTSD, partial PTSD and PTSD-like symptoms, after removing the data for women with PTSD and partial PTSD prior to childbirth, are presented in Table 2. The rates of PTSD at every timepoint postpartum, after removing these data, are below the uncontrolled figure as presented in Table 1 . As can be seen, the rates of PTSD postpartum are lower $(4.7 \%)$ than those initially reported in Table 1. Furthermore, postpartum rates of full PTSD-like symptoms are reduced. Yet, the rates of partial PTSD and partial PTSD-like symptoms are comparable with the rates reported in Table 1.

\section{Clinically significant depression and anxiety during pregnancy}

Approximately 122 women (13.1\%) had scores on the EPDS that were consistent with a probable diagnosis of depression (EPDS score $\geqslant 14$ ) and another 9.1\% $(n=85)$ had scores consistent with possible depression (EPDS score $=12$ or 13 ). In addition, $26.8 \%$ of women $(n=250)$ had clinically significant symptoms of state anxiety and $29.7 \%$ of women $(n=277)$ had clinically significant symptoms of trait anxiety.

\section{Adjusted PTSD, partial PTSD, and PTSD-like symptoms postpartum}

Table 3 presents adjusted estimates of PTSD, partial PTSD and PTSD-like symptoms after removing the data for women with prior PTSD and partial PTSD and clinically significant symptoms of depression or anxiety during pregnancy (as assessed during the last trimester of pregnancy). With a PTSD rate of $3.1 \%$ for women with no history of prior trauma symptomatology or depression or anxiety during pregnancy, it is clear that childbirth events can be traumatic and precipitate the onset of PTSD postpartum.

\section{Traumatic birth events and psychopathology postpartum}

Approximate rates of clinically significant symptoms of postpartum depression and anxiety following a traumatic birth event, with and without concurrent PTSD and partial PTSD, are shown in Table 4. It is evident that, after experiencing a traumatic birth, more women with possible depression had no PTSD classification, in comparison with women with possible depression and experiencing PTSD or partial PTSD. By contrast, there was a comparable number of women with full PTSD and probable depression and women with no PTSD classification but with probable depression (at 12 weeks and 24 weeks postpartum). Also, while women with a PTSD classification experienced significant anxiety, more women with clinically significant anxiety did not have a PTSD classification.

We also assessed for 'other' traumatic events (criteria A1 and A2) between birth and 24 weeks postpartum at the last assessment time-point. The way the time-frame was collected meant that we could assess the incidence of PTSD from childbirth at 12 weeks 
Table 2. Adjusted prevalence of PTSD, partial PTSD and PTSD-like symptoms postpartum after controlling for prior PTSD, partial PTSD and PTSD-like symptoms

\begin{tabular}{lccc}
\hline Postpartum & $4-6$ weeks & 3 months & 6 months \\
\hline PTSD & & & \\
Full PTSD & $18(2.4 \%)$ & $34(4.7 \%)$ & $32(4.7 \%)$ \\
Full PTSD without control & $31(3.6 \%)$ & $52(6.3 \%)$ & $45(5.8 \%)$ \\
Partial PTSD group 1 & $11(1.4 \%)$ & $15(2.1 \%)$ & $11(1.6 \%)$ \\
Partial PTSD group 1 without control & $12(1.4 \%)$ & $17(2.1 \%)$ & $14(1.8 \%)$ \\
Partial PTSD group 2 & $1(0.1 \%)$ & $2(0.3 \%)$ & $0(0.0 \%)$ \\
Partial PTSD group 2 without control & $1(0.1 \%)$ & $2(0.2 \%)$ & $1(0.1 \%)$ \\
Partial (group 3) & $2(0.2 \%)$ & $4(0.5 \%)$ & $2(0.3 \%)$ \\
Partial (group 3) without control & $4(0.5 \%)$ & $6(0.7 \%)$ & $2(0.3 \%)$ \\
PTSD-like symptoms & & & \\
Full PTSD-like symptoms & $3(0.4 \%)$ & $17(2.3 \%)$ & $16(2.3 \%)$ \\
Full PTSD-like symptoms without control & $4(0.5 \%)$ & $23(2.8 \%)$ & $23(3.0 \%)$ \\
Partial PTSD-like symptoms group 1 & $5(0.7 \%)$ & $11(1.5 \%)$ & $5(0.7 \%)$ \\
Partial PTSD-like group 1 without control & $6(0.7 \%)$ & $13(1.6 \%)$ & $5(0.6 \%)$ \\
Partial PTSD-like symptoms group 2 & $0(0.0 \%)$ & $0(0.0 \%)$ & $0(0.0 \%)$ \\
Partial PTSD-like group 2 without control & $0(0.0 \%)$ & $0(0.0 \%)$ & $0(0.0 \%)$ \\
Partial PTSD-like symptoms group 3 & $0(0.0 \%)$ & $2(0.3 \%)$ & $0(0.0 \%)$ \\
Partial PTSD-like group 3 without control & $0(0.0 \%)$ & $3(0.4 \%)$ & $0(0.0 \%)$ \\
No classification & $722(94.8 \%)$ & $643(88.3 \%)$ & $620(90.4 \%)$ \\
No classification without control & $808(93.3 \%)$ & $710(86.0 \%)$ & $686(88.4 \%)$ \\
Total no. (\%) & $762(100 \%)$ & $728(100 \%)$ & $686(100 \%)$ \\
Total no. (\%) & $866(100 \%)$ & $826(100 \%)$ & $776(100 \%)$ \\
\hline & & & \\
\hline
\end{tabular}

Partial post-traumatic stress disorder (PTSD) group 1 met all PTSD criteria except for one or two of the necessary three criterion $C$ symptoms; group 2 met all PTSD criteria except for one of the necessary two criterion D symptoms; group 3 met all PTSD criteria except for one or two of the necessary three criterion C symptoms and one of the necessary two criterion D symptoms. Partial PTSD-like symptom groups met the same criteria as partial PTSD except the criteria for a traumatic birth event were not met (i.e. a non-criterion A event).

postpartum and women who endorsed having experienced another traumatic event between birth and 12 weeks. This cross tabulation was statistically significant, although with a small effect $\left[\chi^{2}(1), n=826\right)=$ $8.48, p<0.01, \varphi=0.1]$.

\section{Non-traumatic birth events and psychopathology postpartum}

Table 5 presents the rates of clinically significant symptoms of postpartum depression and anxiety following a non-traumatic birth event. Rates of concurrent PTSD-like symptoms are also presented. As indicated in the table, most women with possible depression did not have a PTSD-like symptom classification. Yet, more women with probable depression, in comparison with women with possible depression, had a full PTSD-like symptom classification, although this could reflect general distress postpartum. Likewise, a number of women who had clinically significant symptoms of state and trait anxiety also had a
PTSD-like symptom classification. Again, this may be reflective of general distress following childbirth.

\section{Discussion and recommendations}

The current study provides data on PTSD in pregnancy and childbirth that can be considered representative of actual occurrence. Approximately 91\% of women self-reported experiencing a potentially traumatic event at some point in their life, which is similar to that reported in other trauma research (e.g. Stein et al. 2000; Freedman et al. 2002). Also, 43.3\% of the sample met criteria for a traumatic event, which is also consistent with past epidemiological research (e.g. Kessler et al. 1995; Creamer et al. 2001). A considerable number of women had experienced two $(n=187,22.2 \%)$ or more $(n=448,53.1 \%)$ potentially traumatic events throughout their life; again, similar rates to those reported in epidemiological research studies (e.g. Creamer et al. 2001). The rate of PTSD 
Table 3. Adjusted prevalence of PTSD, partial PTSD and PTSD-like symptoms postpartum after controlling for prior PTSD phenomenology and clinically significant depression and anxiety during pregnancy

\begin{tabular}{lrrr}
\hline Postpartum & $4-6$ weeks & 3 months & 6 months \\
\hline PTSD & & & \\
Full PTSD & $6(1.2 \%)$ & $15(3.1 \%)$ & $14(3.1 \%)$ \\
Partial PTSD group 1 & $5(1.0 \%)$ & $8(1.7 \%)$ & $6(1.3 \%)$ \\
Partial PTSD group 2 & $1(0.2 \%)$ & $1(0.2 \%)$ & $0(0.0 \%)$ \\
Partial PTSD group 3 & $2(0.4 \%)$ & $2(0.4 \%)$ & $0(0.0 \%)$ \\
PTSD-like symptoms & & & \\
Full PTSD-like symptoms & $3(0.6 \%)$ & $8(1.7 \%)$ & $8(1.8 \%)$ \\
Partial PTSD-like symptoms group 1 & $4(0.8 \%)$ & $10(2.1 \%)$ & $2(0.4 \%)$ \\
Partial PTSD-like symptoms group 2 & $0(0.0 \%)$ & $0(0.0 \%)$ & $0(0.0 \%)$ \\
Partial PTSD-like symptoms group 3 & $0(0.0 \%)$ & $1(0.2 \%)$ & $0(0.0 \%)$ \\
No classification & $479(95.8 \%)$ & $432(90.6 \%)$ & $423(93.4 \%)$ \\
Total no. (\%) & $500(100 \%)$ & $477(100 \%)$ & $453(100 \%)$ \\
& & & \\
\hline
\end{tabular}

Partial post-traumatic stress disorder (PTSD) group 1 met all PTSD criteria except for one or two of the necessary three criterion $\mathrm{C}$ symptoms; group 2 met all PTSD criteria except for one of the necessary two criterion D symptoms; group 3 met all PTSD criteria except for one or two of the necessary three criterion C symptoms and one of the necessary two criterion D symptoms. Partial PTSD-like symptom groups met the same criteria as partial PTSD except the criteria for a traumatic birth event were not met (i.e. a non-criterion A event).

before childbirth was found to be $8.0 \%$ of women, similar to that found by Kessler et al. (1995), who reported a lifetime prevalence of PTSD equal to $7.8 \%$. We are, therefore, satisfied that our intake sample was reasonably representative of pregnant women in the community.

The current study also revealed that nearly half of women experienced a traumatic birth event. However, in comparison with the number of traumatic birth events, a much smaller percentage of women developed PTSD postpartum. That $45.5 \%$ of women reported, between 4 and 6 weeks after the birth, as having met PTSD criteria A1 (experienced threat to self or baby) and A2 (experienced fear, helplessness or horror) during the birth is worthy of comment. In epidemiological surveys, $50 \%$ of women and $65 \%$ of men have experienced a traumatic event excluding birth (A1; Creamer et al. 2001) and, of those who endorse A1, $81 \%$ of females and $69 \%$ of males also endorse A2 (Creamer et al. 2005). Our study demonstrated lower A1 and A2 concordance rates, with only $54.2 \%$ of those women endorsing A1 also endorsing A2. This lower concordance rate may be due to the expectation or interpretation of birth being a dangerous time for mother and baby regardless of subjective fear, helplessness or horror. However, with only $8.16 \%$ of women who had endorsed A2 not endorsing A1, it appears that subjective fear without viewing the birth as dangerous is unusual.
In the Creamer et al. (2005) study, of those who endorsed A2, 97\% went on to endorse persistent traumatic memories of the event. In deriving the DSM-IV-TR criteria (APA, 2000), the intention may have been to have both objective (A1) and subjective (A2) appraisals of event status (traumatic versus nontraumatic). However, criterion A1, as it currently stands, still requires a subjective interpretation of the event.

In the current study, we asked whether the woman interpreted the birth in a similar fashion as if we were asking a returned servicewoman or any other person about a critical event. We had no doubts that the woman had given birth. That this could instigate pause for concern from researchers in the field suggests an a priori worry with criterion creep (or bracket creep; McNally, 2003) regarding whether birth could possibly be seen as traumatic at an objective (i.e. subjective third party) level. We caution against such an approach, with the preponderance of research conducted into the predictive value of A1 events finding paradoxical outcomes with 'non-traumatic' or 'stressful life' events being associated with higher trauma symptomatology than classical 'traumatic' events (Long et al. 2008; van Hooff et al. 2009). That just under half of our sample endorsed criteria A1 and A2 suggests that women who have given birth a month earlier either view the birth as dangerous and causing fear and/or have a lowered threshold for what 
Table 4. Rates of clinically significant symptoms of depression and anxiety postpartum following a traumatic birth event with and without PTSD and partial PTSD

\begin{tabular}{|c|c|c|c|}
\hline Postpartum & 4-6 weeks & 3 months & 6 months \\
\hline \multicolumn{4}{|l|}{ Possible depression } \\
\hline With full PTSD & $8(16.0 \%)$ & $6(12.5 \%)$ & $10(26.3 \%)$ \\
\hline With partial PTSD group 1 & $2(4.0 \%)$ & $6(12.5 \%)$ & $1(2.6 \%)$ \\
\hline With partial PTSD group 2 & $0(0.0 \%)$ & $1(2.1 \%)$ & $0(0.0 \%)$ \\
\hline With partial PTSD group 3 & $0(0.0 \%)$ & $1(2.1 \%)$ & $1(2.6 \%)$ \\
\hline No PTSD classification & $40(80.0 \%)$ & $34(70.8 \%)$ & $26(68.4 \%)$ \\
\hline Total no. $(\%)$ & $50(100 \%)$ & $48(100 \%)$ & $38(100 \%)$ \\
\hline \multicolumn{4}{|l|}{ Probable depression } \\
\hline With full PTSD & $19(27.9 \%)$ & $37(47.4 \%)$ & $30(39.0 \%)$ \\
\hline With partial PTSD group 1 & $3(4.4 \%)$ & $4(5.1 \%)$ & $3(3.9 \%)$ \\
\hline With partial PTSD group 2 & $1(1.5 \%)$ & $0(0.0 \%)$ & $0(0.0 \%)$ \\
\hline With partial PTSD group 3 & $0(0.0 \%)$ & $0(0.0 \%)$ & $0(0.0 \%)$ \\
\hline No PTSD classification & $45(66.2 \%)$ & $37(47.4 \%)$ & $44(57.1 \%)$ \\
\hline Total no. (\%) & $68(100 \%)$ & $78(100 \%)$ & $77(100 \%)$ \\
\hline \multicolumn{4}{|c|}{ Clinically significant state anxiety } \\
\hline With full PTSD & $18(21.2 \%)$ & $34(34.3 \%)$ & $27(30.0 \%)$ \\
\hline With partial PTSD group 1 & $3(3.5 \%)$ & $7(7.1 \%)$ & $4(4.4 \%)$ \\
\hline With partial PTSD group 2 & $1(1.2 \%)$ & $0(0.0 \%)$ & $1(1.1 \%)$ \\
\hline With partial PTSD group 3 & $0(0.0 \%)$ & $1(1.0 \%)$ & $1(1.1 \%)$ \\
\hline No PTSD classification & $63(74.1 \%)$ & $57(57.6 \%)$ & $57(63.3 \%)$ \\
\hline Total no. (\%) & $85(100 \%)$ & 99 (100) & $90(100 \%)$ \\
\hline \multicolumn{4}{|c|}{ Clinically significant trait anxiety } \\
\hline With full PTSD & $25(18.9 \%)$ & $45(32.8 \%)$ & $36(28.8 \%)$ \\
\hline With partial PTSD group 1 & $7(5.3 \%)$ & $11(8.0 \%)$ & $7(5.6 \%)$ \\
\hline With partial PTSD group 2 & $1(0.8 \%)$ & $1(0.7 \%)$ & $1(0.8 \%)$ \\
\hline With partial PTSD group 3 & $1(0.8 \%)$ & $2(1.5 \%)$ & $1(0.8 \%)$ \\
\hline No PTSD classification & $98(74.2 \%)$ & $78(56.9 \%)$ & $80(64.0 \%)$ \\
\hline Total no. (\%) & $132(100 \%)$ & $137(100 \%)$ & $125(100 \%)$ \\
\hline
\end{tabular}

Partial post-traumatic stress disorder (PTSD) group 1 met all PTSD criteria except for one or two of the necessary three criterion $C$ symptoms; group 2 met all PTSD criteria except for one of the necessary two criterion D symptoms; group 3 met all PTSD criteria except for one or two of the necessary three criterion C symptoms and one of the necessary two criterion D symptoms.

constitutes danger and fear. The need for a distinction between these two interpretations has not been proven. Whether A1 and A2 related to childbirth have external, long-term validity (i.e. related to functional impairment and help-seeking behaviours due to memories of the birth), as well as short-term, internal validity (predicting PTSD criteria B, C and D) remains to be seen.

Women who developed PTSD as a result of childbirth invariably had experienced an unexpected and potentially harrowing obstetric event. This finding differs from previous findings (Ballard et al. 1995), where most women with a profile of PTSD had experienced a normal vaginal delivery. We believe that a more exhaustive history, taking assessment of the birthing experience, may be partially responsible for this difference in results.

Consistent with other research findings (Ballard et al. 1995), this study also found that diagnoses of PTSD postpartum increased initially over time and then appeared to stabilize. However, unlike past research, we found that total PTSD symptom severity decreased initially postpartum and then appeared relatively stable. Similarly, the findings indicated that women can experience clinically significant levels of depression and anxiety postpartum and these rates are comparable with rates obtained in other research studies (Brown \& Lumley, 1998).

We also found that women who obtained a diagnosis of PTSD at 12 weeks postpartum were more 
Table 5. Rates of clinically significant symptoms of depression and anxiety postpartum following a non-traumatic birth event with and without PTSD-like symptoms

\begin{tabular}{lccc}
\hline Postpartum & $4-6$ weeks & 3 months & 6 months \\
\hline Possible depression & & & \\
With full PTSD-like symptoms & $1(2.6 \%)$ & $3(6.8 \%)$ & $4(7.5 \%)$ \\
With partial PTSD-like symptoms (group 1) & $1(2.6 \%)$ & $1(2.3 \%)$ & $3(5.7 \%)$ \\
No PTSD-like classification & $37(94.9 \%)$ & $40(90.9 \%)$ & $46(86.8 \%)$ \\
Total no. (\%) & $39(100 \%)$ & $44(100 \%)$ & $53(100 \%)$ \\
Probable depression & & & \\
With full PTSD-like symptoms & $3(8.8 \%)$ & $13(24.5 \%)$ & $15(34.9 \%)$ \\
With partial PTSD-like symptoms group 1 & $1(2.9 \%)$ & $2(3.8 \%)$ & $0(0.0 \%)$ \\
No PTSD-like symptoms classification & $30(88.2 \%)$ & $38(71.7 \%)$ & $28(65.1 \%)$ \\
Total no. (\%) & $34(100 \%)$ & $53(100 \%)$ & $43(100 \%)$ \\
Clinically significant state anxiety & & & \\
With full PTSD-like symptoms & $1(2.1 \%)$ & $11(18.0 \%)$ & $11(17.7 \%)$ \\
With partial PTSD-like symptoms group 1 & $0(0.0 \%)$ & $1(1.6 \%)$ & $2(3.2 \%)$ \\
No PTSD-like symptoms classification & $46(97.9 \%)$ & $49(80.3 \%)$ & $49(79.0 \%)$ \\
Total no. (\%) & $47(100 \%)$ & $61(100 \%)$ & $62(100 \%)$ \\
Clinically significant trait anxiety & & & \\
With full PTSD-like symptoms & $2(2.7 \%)$ & $16(16.2 \%)$ & $20(20.8 \%)$ \\
With partial PTSD-like symptoms group 1 & $1(1.4 \%)$ & $3(3.0 \%)$ & $1(1.0 \%)$ \\
With partial PTSD-like symptoms group 3 & $0(0.0 \%)$ & $1(1.0 \%)$ & $0(0.0 \%)$ \\
No PTSD-like symptoms classification & $71(95.9 \%)$ & $79(79.8 \%)$ & $75(78.1 \%)$ \\
Total no. (\%) & $74(100 \%)$ & $99(100 \%)$ & $96(100 \%)$ \\
& & &
\end{tabular}

Partial post-traumatic stress disorder (PTSD)-like symptoms group 1 met all PTSD criteria except for criteria A and one or two of the necessary three criterion C symptoms; partial PTSD-like symptoms group 2 met all PTSD criteria except for criteria A and one of the necessary two criterion D symptoms; partial PTSD-like symptoms group 3 met all PTSD criteria except for criteria A and one or two of the necessary three criterion $\mathrm{C}$ symptoms and one of the necessary two criterion $\mathrm{D}$ symptoms.

likely to experience and/or endorse other events experienced between birth and 12 weeks postpartum as 'traumatic'. However, it should be stressed that to obtain a diagnosis of PTSD from birth, the woman answered the criteria related solely in relation to the birth and we do not know whether the women who endorsed experiencing another traumatic event would have met the diagnostic criteria of PTSD from that event.

The method utilized during the present study improves on prior research that examined PTSD postpartum by having a large sample, a validated measure of PTSD, assessing impairment and accounting for pre-existent PTSD, depression and anxiety. This approach permits stronger conclusions to be drawn regarding the prevalence of PTSD following childbirth. However, we suggest that while the rates in the present study were compared with those identified through the CAPS, structured clinical interviews for all Axis I disorders (e.g. SCID) may lead to improved specificity in future research.
While this study utilized self-report measures, structured diagnostic interviews were also used, with good agreement between the CAPS and the PDS. Due to the chronicity of untreated PTSD (Sommer \& Ehlert, 2004), it would also be advantageous to follow up our current sample for longer than 12 months and assess outcome over at least a 5-year time-span, offering treatment to those with a continued disorder. Considering the attrition rate was over-represented by people from a lower SES and who initially displayed more pathology, these results would, if anything, represent an underestimate of total pathology and impairment.

The findings that a large number of women perceive childbirth to be traumatic, and that approximately $5.8 \%$ of women $(3.1 \%$ when removing any women with a history of PTSD, anxiety or depression during pregnancy) will still have diagnosable PTSD at 6 months postpartum, speaks to the need for additional research into traumatic childbirth events and PTSD postpartum. In 2003, 251200 births were 
registered in Australia (Australian Bureau of Statistics, 2004), 695500 were registered in the UK (National Statistics Online, 2007) and in the USA 4.12 million births were registered in 2004 (Centres for Disease Control and Prevention, 2006). Thus, traumatic birth events and PTSD could potentially impact on a significant number of women worldwide. Overall, these data suggest the need for healthcare professionals and researchers to assess the nature of childbirth events and anxiety conditions, including PTSD, postpartum. Such research could inform prevention and treatment efforts.

\section{Acknowledgements}

Funding for this research: 2003-2005, Ipswich Hospital Foundation Grant. All authors had full access to all of the data in the study and take responsibility for the integrity of the data and the accuracy of the data analysis.

\section{Declaration of Interest}

None.

\section{References}

Allen S (1998). A qualitative analysis of the process, mediating variables and impact of traumatic childbirth Journal of Reproductive and Infant Psychology 16, 107-131.

APA (2000). Diagnostic and Statistical Manual of Mental Disorders, 4 th edn - text revision. American Psychiatric Association: Washington, DC.

Australian Bureau of Statistics (2004). Births, Australia, No. 3301.0. Australian Bureau of Statistics: Canberra, Australian Capital Territory.

Australian Bureau of Statistics (2007). Household Income and Income Distribution, Australia, 2005-06. Australian Bureau of Statistics: Canberra, ACT.

Ayers S (2001). Assessing psychopathology in pregnancy and postpartum. Journal of Psychosomatic Obstetrics and Gynecology 22, 91-102.

Ayers S, Pickering AD (2001). Do women get posttraumatic stress disorder as a result of childbirth? A prospective study of incidence. Birth: Issues in Perinatal Care $\mathbf{2 8}$, 111-118.

Bailham D, Joseph S (2003). Post-traumatic stress following childbirth: a review of the emerging literature and directions for research and practice. Psychology, Health and Medicine 8, 159-168

Baldwin S, Williams D, Houts A (2004). The creation, expansion, and embodiment of Posttraumatic Stress Disorder: a case study in historical critical psychopathology. Scientific Review of Mental Health Practice 3, 33-52.

Ballard CG, Stanley AK, Brockington IF (1995). Post-traumatic stress disorder (PTSD) after childbirth British Journal of Psychiatry 166, 525-528.
Ballenger JC, Davidson J, Lecrubier Y, Nutt DJ, Foa E, Kessler RC, McFarlane AC, Shalev AY, (2000). Consensus statement on posttraumatic stress disorder from the international consensus group on depression and anxiety. Journal of Clinical Psychiatry 61, 60-66.

Beck C (2004a). Post-traumatic stress disorder due to childbirth: the aftermath. Nursing Research 53, 216-224.

Beck C (2004b). Birth trauma: in the eye of the beholder. Nursing Research 53, 28-35.

Brown S, Lumley J (1998). Maternal health after childbirth: results of an Australian population based survey. British Journal of Obstetrics and Gynaecology 105, 156-161.

Centres for Disease Control and Prevention (2006). Births: final data for 2004. National Vital Statistics Reports 55, 1-102.

Cox JL, Holden J, Sagovsky R (1987). Detection of postnatal depression: development of the 10-item Edinburgh Postnatal Depression Scale. British Journal of Psychiatry 150, 782-786.

Creamer M, Burgess P, McFarlane AC (2001). Post-traumatic stress disorder: findings from the Australian National Survey of Mental Health and Well-being. Psychological Medicine 31, 1237-1247.

Creamer M, McFarlane AC, Burgess P (2005). Psychopathology following trauma: the role of subjective experience. Journal of Affective Disorders 86, 175-782.

Creedy DK, Shochet IM, Horsfall J (2000). Childbirth and the development of acute trauma symptoms: incidence and contributing factors. Birth 27, 104-111.

Eberhard-Gran M, Eskild A, Tambs K, Opjordsmoen S, Samuelsen SO (2001). Review of validation studies of the Edinburgh Postnatal Depression Scale. Acta Psychiatrica Scandinavica 104, 243-249.

Engelhard IM, van den Hout MA, Kindt M (2003). The relationship between neuroticism, pre-traumatic stress, and post-traumatic stress: a prospective study. Personality and Individual Differences 35, 381-388.

Foa E, Cashman L, Jaycox L, Perry K (1997). The validation of a self-report measure of posttraumatic stress disorder: the Posttraumatic Diagnostic Scale. Psychological Assessment 9, $445-451$.

Foa E, Meadows E (1997). Psychosocial treatments for posttraumatic stress disorder: a critical review. Annual Review of Psychology 48, 449-480.

Fones C (1996). Posttraumatic stress disorder occurring after painful childbirth. Journal of Nervous and Mental Disease 184, 195-196.

Freedman SA, Gluck N, Tuval-Mashiach R, Brandes D, Peri T, Shalev A (2002). Gender differences in responses to traumatic events: a prospective study. Journal of Traumatic Stress 15, 407-413.

Greening L, Stoppelbein L, Docter R (2002). The mediating effects of attributional style and event-specific attributions on post-disaster adjustment. Cognitive Therapy and Research 26, 261-274.

Griffin MG, Uhlmansiek MH, Resick PA, Mechanic MB (2004). Comparison of the Posttraumatic Stress Disorder Scale versus the Clinician-Administered Posttraumatic Stress Disorder Scale in Domestic Violence Survivors. Journal of Traumatic Stress 17, 497-503. 
Johnston-Robledo I, Barnack J (2004). Psychological issues in childbirth: potential roles for psychotherapists. Women and Therapy 27, 133-150.

Keogh E, Ayers S, Francis H (2002). Does anxiety sensitivity predict post-traumatic stress symptoms following childbirth? A preliminary report. Cognitive Behaviour Therapy 31, 145-155.

Kessler RC, Sonnega A, Bromet E, Hughes M, Nelson CB (1995). Posttraumatic stress disorder in the National Comorbidity Survey. Archives of General Psychiatry 52, 1048-1060.

Laws P, Hilder L (2008). Australia's Mothers and Babies 2006. Australian Institute of Health and Welfare: Sydney, NSW.

Long ME, Elhai JD, Schweinle A, Gray MJ, Grubaugh AL, Frueh C (2008). Differences in posttraumatic stress disorder diagnostic rates and symptom severity between criterion A1 and non-criterion A1 stressors. Journal of Anxiety Disorders 22, 1255-1263.

McNally RJ (2003). Progress and controversy in the study of posttraumatic stress disorder. Annual Review of Psychology 54, 229-252.

Matthey S (2004). Calculating clinically significant change in postnatal depression studies using the Edinburgh Postnatal Depression Scale. Journal of Affective Disorders 78, 269-272.

Matthey S, Barnett B, Howie P, Kavanagh DJ (2003). Diagnosing postpartum depression in mothers and fathers: whatever happened to anxiety? Journal of Affective Disorders 74, 139-147.

Murray D, Cox J (1990). Screening for depression during pregnancy with the Edinburgh Depression Scale (EPDS). Journal of Reproductive and Infant Psychology 8, 99-107.

National Statistics Online (2007). UK Office of National Statistics: people and migration - births and deaths (http:/ / www.statistics.gov.uk/cci/nugget.asp?id=761). Accessed 27 November 2007.

Olde E, van der Hart O, Kleber RJ, van Son MJ, Wijnen HA, Pop VJ (2005). Peritraumatic dissociation and emotions as predictors of PTSD symptoms following childbirth. Journal of Trauma and Dissociation 6, 125-142.

Righetti-Veltema M, Conne-Perreard E, Bousquet A, Manzano J (2002). Postpartum depression and mother-infant relationship at 3 months old. Journal of Affective Disorders 70, 291-306.
Ryding E, Persson A, Onell C, Kvist L (2003). An evaluation of midwives' counselling of pregnant women in fear of childbirth. Acta Obstetricia et Gynecologica Scandinavica 82, 10-17.

Saisto T, Salmela-Aro K, Nurmi JE, Kononen T, Halmesmaki E (2001). A randomized controlled trial of intervention in fear of childbirth. Obstetrics and Gynecology 98, 820-826.

Sjostrom K, Valentin L, Thelin T, Marsal K (2002). Maternal anxiety in late pregnancy: effect on fetal movements and fetal heart rate. Early Human Development 67, 87-100.

Social Policy Research Centre (2006). Baseline Demographic Profile 2001. Social Policy Research Centre: Sydney, NSW.

Söderquist J, Wijma B, Wijma K (2006). The longitudinal course of post-traumatic stress after childbirth. Journal of Psychosomatic Obstetrics and Gynaecology 27, 113-119.

Soet JE, Brack GA, Dilorio C (2003). Prevalence and predictors of women's experience of psychological trauma during childbirth. Birth 30, 36-46.

Sommer I, Ehlert U (2004). Adjustment to trauma exposure: prevalence and predictors of posttraumatic stress disorder symptoms in mountain guides. Journal of Psychosomatic Research 57, 329-335.

Spielberger C, Gorsuch R, Lushene R, Vagg P, Jacobs G (1983). Manual for the State-Trait Anxiety Inventory (Form Y). Consulting Pscychologists Press, Inc: Palo Alto, CA.

Stein MB, Walker JR, Forde DR (2000). Gender differences in susceptibility to posttraumatic stress disorder. Behaviour Research and Therapy 38, 619-628.

Stein MB, Walker JR, Hazen AL, Forde DR (1997). Full and partial posttraumatic stress disorder: findings from a community survey. American Journal of Psychiatry 154, 1114-1119.

Switzer GE, Dew MA, Thompson K, Goycoolea JM, Derricott T, Mullins SD (1999). Posttraumatic stress disorder and service utilization among urban mental health center clients. Journal of Traumatic Stress 12, 25-39.

van Hooff M, McFarlane AC, Baur J, Abraham M, Barnes DJ (2009). The stressor Criterion-A1 and PTSD: a matter of opinion? Journal of Anxiety Disorders 23, 77-86.

Wijma K, Soderquist J, Wijma B (1997). Posttraumatic stress disorder after childbirth: a cross sectional study. Journal of Anxiety Disorders 11, 587-597. 